\title{
Structural and chemical alteration of crystalline olivine under low energy $\mathrm{He}^{+}$irradiation
}

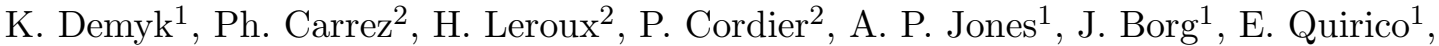 \\ P. I. Raynal ${ }^{1}$, and L. d'Hendecourt ${ }^{1}$
}

1 IAS-CNRS, "Astrochimie Expérimentale", Université Paris XI, Bâtiment 121, 91405 Orsay Cedex, France

${ }^{2}$ LSPES, ESA CNRS 8008, Université Sciences et Technologies de Lille, 59655 Villeneuve d'Ascq Cedex, France

Received 18 December 2000 / Accepted 6 February 2001

\begin{abstract}
We present the results of irradiation experiments on crystalline olivine with $\mathrm{He}^{+}$ions at energies of 4 and $10 \mathrm{keV}$ and fluences varying from $510^{16}$ to $10^{18}$ ions $/ \mathrm{cm}^{2}$. The aim of these experiments is to simulate ion implantation into interstellar grains in shocks in the ISM. Irradiated samples were analysed by transmission electron microscopy (TEM). The irradiation causes the amorphization of the olivine, at all $\mathrm{He}^{+}$fluences considered. The thickness of the amorphized region is $40 \pm 15 \mathrm{~nm}$ and $90 \pm 10 \mathrm{~nm}$ for the $4 \mathrm{keV}$ and $10 \mathrm{keV}$ experiments, respectively. The amorphization of the olivine occurs in conjunction with an increase in the porosity of the material due to the formation of bubbles. In addition, the amorphized layer is deficient in oxygen and magnesium. We find that the $\mathrm{O} / \mathrm{Si}$ and $\mathrm{Mg} / \mathrm{Si}$ ratios decrease as the $\mathrm{He}^{+}$fluence increases. These experiments show that the irradiation of dust in supernova shocks can efficiently alter the dust structure and composition. Our result are consistent with the lack of crystalline silicates in the interstellar medium and also with the compositional evolution observed from olivine-type silicates around evolved stars to pyroxene-type silicates around protostars.
\end{abstract}

Key words. methods: laboratory - ISM: dust, extinction, evolution - shock waves

\section{Introduction}

The results from the Infrared Space Observatory (ISO) have raised several questions about the evolution of the structure and chemical composition of silicate dust. The ISO-SWS spectra $(2-45 \mu \mathrm{m})$ of some evolved oxygen-rich stars exhibit emission bands at wavelengths $\geq 10 \mu \mathrm{m}$ (e.g. Waters et al. 1998). These bands are due to lattice vibrations in crystalline silicates formed in situ around these stars. They are not present in the spectra of disordered silicates which show only two broad and structureless bands at $\sim 9.7$ and $18 \mu \mathrm{m}$. This newly-formed dust is injected in the interstellar medium (ISM) via stellar winds. However, these crystalline silicate bands have not been detected, in emission or absorption, in the ISM or in class 0 protostellar objects (Demyk 2000). This could be explained if the spectral signatures of the crystalline silicates are undetectable in the ISM spectra. Indeed, crystalline silicate are not observed in low mass-loss rate AGB stars (Cami et al. 1998; Sylvester et al. 1999) and may thus not be produced in such stars (Sogawa \& Kozasa 1999). Thus, newly-formed crystalline silicates could be "diluted" in the ISM by amorphous silicate components produced in low, and also high, mass-loss rate stars. If this dilution

Send offprint requests to: K. Demyk, e-mail: demyk@ias.fr is sufficient, the crystalline silicates may not be abundant enough to be detectable in ISM spectra. However, some crystalline silicates could be present around low-mass loss rate stars and their emission bands be undetectable because of radiative transfer effects (Kemper et al. 2000). In that case, the dilution effect may not be sufficient to explain the absence of the bands in the ISM. The disappearance of the crystalline silicates may then be due to their destruction, or to the amorphization of their crystalline structure, in the ISM. The selective destruction of crystalline silicates is unlikely and we therefore consider that their amorphization in the ISM is the most probable explanation.

Silicates around evolved stars are usually identified with olivine-type silicates. Some pyroxenes are present around these stars in the form of crystalline enstatite and/or diopside but its abundance generally does not exceed $\sim 10-15 \%$ of the silicate dust mass (Waters et al. 1998; Demyk et al. 2000). In molecular clouds and in protostellar spectra, the Si-O stretching mode of the silicates is shifted to slightly shorter wavelengths than in the spectra of evolved stars and is better reproduced with pyroxene-type silicates (Dorschner et al. 1988; Bowey et al. 1998; Demyk et al. 1999). Thus, in addition to a change of structure, the chemical composition of the interstellar silicates seems to vary from evolved stars to protostars. 
The evolution of the structural and chemical nature of the silicate dust certainly occurs in the ISM where the dust is subjected to processing, e.g., grain-grain and gasgrain collisions, interactions with photons and cosmic rays, and shocks (e.g. Jones 2000; Jones et al. 1996). Among these processes, energetic interactions with ions can induce the amorphization of crystalline minerals and change their chemical composition. Experiments on the irradiation of crystalline olivine with high energy protons, typical of cosmic rays, show no alteration of the crystalline structure at fluences relevant to the ISM (Day 1977). However, in supernovae shocks, grains interact with the atoms accelerated and ionised by the shock wave. The energies of these ions, which depend on the shock velocity, are of the order of keV. Several studies show that it is possible to amorphize olivine and pyroxene with $\mathrm{H}^{+}$and $\mathrm{He}^{+}$ions with energies in the $4-20 \mathrm{keV}$ range (Borg et al. 1979; Borg 1982; Bradley 1994). Furthermore, chemical changes were observed in the composition of olivine irradiated with $20 \mathrm{keV} \mathrm{H}^{+}$(Bradley 1994) and $1 \mathrm{keV} \mathrm{H}^{+}$and $4 \mathrm{keV} \mathrm{He}{ }^{+}$ ions (Dukes et al. 1999).

In this letter, we present the results of new experiments on the irradiation of crystalline olivine aimed at simulating the interaction between the grains and the accelerated and ionised gas in supernovae shocks. The experiments and analysis of the irradiated samples are described in Sect. 2. The results and the astrophysical implications are presented in Sects. 3 and 4, respectively.

\section{Experiments}

In order to obtain a high surface/volume ratio, crystalline San Carlos olivine single crystals $\left(\mathrm{Mg}_{1.8} \mathrm{Fe}_{0.2} \mathrm{SiO}_{4}\right)$ were prepared using classical TEM sample preparation procedure, i.e. ion-thinned by low angle of incidence $\mathrm{Ar}^{+}$bombardment. The final samples have a bevelled shape (Fig. 1) whose point is characterised by a high surface/volume ratio, comparable to that of interstellardust.

The samples were irradiated at the CSNSM facility (Orsay) with $\mathrm{He}^{+}$ions accelerated to energies of 4 and $10 \mathrm{keV}$. Several samples were irradiated at $4 \mathrm{keV}$ with different fluences: $510^{16}, 10^{17}, 510^{17}$ and $10^{18} \mathrm{He}^{+} / \mathrm{cm}^{2}$. At $10 \mathrm{keV}$ the fluence was $10^{18} \mathrm{He}^{+} / \mathrm{cm}^{2}$. The irradiations were performed under vacuum at a pressure of $10^{-7} \mathrm{mbar}$ and at room temperature. In order to avoid excessive heating of the samples during the experiments, the beam current density was maintained below $\sim 9 \mu \mathrm{A} / \mathrm{cm}^{2}$. Furthermore, the analysis of a non-irradiated ion-thinned sample shows that, during the sample preparation, $\mathrm{Ar}^{+}$ bombardment does not alter the structure or the composition of the sample. Thus, the observed changes in the irradiated samples are not to due sample preparation effects but only to the effects of the irradiation.

Analysis of the irradiated samples was carried out by transmission electron microscopy (TEM), with a Philips CM 30 electron microscope operating at $300 \mathrm{keV}$, and equiped with an X-ray energy dispersive spectrometer (EDS Noran-Voyager) having a Ge detector and an ul-

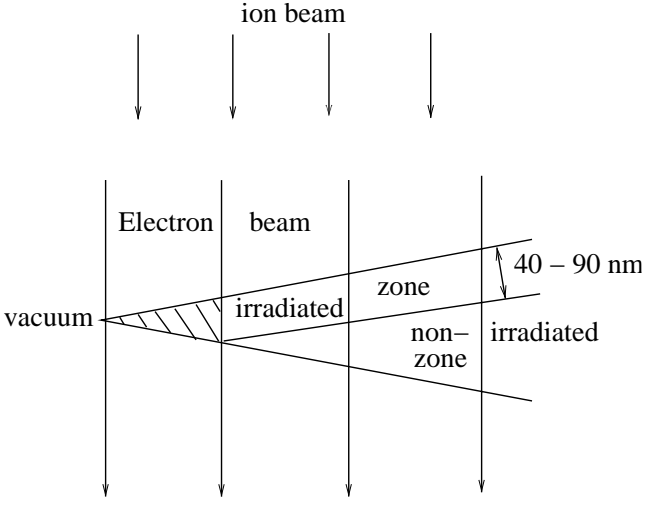

Fig. 1. Experimental configuration for sample irradiation and analysis. The dashed area at the end of the bevelled edge, seen in section, is transparent to the ions and is thus homogeneously irradiated. For thicker regions the ions are stopped in the sample which is then only partially irradiated

tra thin window to allow detection of low-mass elements (e.g. O) with a good sensitivity. Special care was taken during the TEM analysis to derive the thickness of the analysed region and to take into account the possible alteration of the samples under the action of the electron beam. The experimental procedure used for the thickness determination will be described in Carrez et al. (in prep.). The structural and chemical evolution of olivine under the electron beam is presented in Carrez et al. (2001).

\section{Results}

Figure 2 shows an image of the sample irradiated with $10^{18} \mathrm{He}^{+}$ions $/ \mathrm{cm}^{2}$ at $10 \mathrm{keV}$. In thick areas of the sample (right part of Fig. 2), we observe the presence of dark Bragg lines which are due to the diffraction of the incident electrons by the crystalline structure in the sample. In thin areas (left part of the sample in Figs. 1 and 2), where the sample is ion-transparent, the diffraction Bragg lines are not present, indicating that it is completely amorphous. The amorphous structure is confirmed by the diffraction pattern obtained from this region, shown in the inset in Fig. 2. The observed diffuse halo is characteristic of an amorphous material. Similar diffraction patterns were obtained for the samples irradiated at $4 \mathrm{keV}$, indicating that the olivine is amorphized for all fluences. The measured thickness of the amorphized layer is $90 \pm 10 \mathrm{~nm}$ with the $10 \mathrm{keV} \mathrm{He}{ }^{+}$and of $40 \pm 15 \mathrm{~nm}$ with the $4 \mathrm{keV} \mathrm{He}^{+}$.

The implantation of helium ions into the mineral causes bubbles of helium or vacuum, as seen in Fig. 2, which are not present in the non-irradiated sample. For the smallest irradiation fluences, the bubbles are small and the surface of the sample appears like an "orange skin". At a fluence of $10^{18}$ ions $/ \mathrm{cm}^{2}$, the size of the bubbles ranges from $\sim 10 \mathrm{~nm}$ to $\sim 400 \mathrm{~nm}$, with most bubbles of $\sim 50 \mathrm{~nm}$ size. We note that the bubble size increases with fluence.

For each fluence and each energy, we have studied the changes in the sample stoichiometry induced by the irradiation. Figure 3 shows the value of the $\mathrm{O} / \mathrm{Si}$ and $\mathrm{Mg} / \mathrm{Si}$ 
Table 1. Atomic ratio $\mathrm{O} / \mathrm{Si}$ and $\mathrm{Mg} / \mathrm{Si}$ of the irradiated samples

\begin{tabular}{cc|cccc|c}
\hline \hline $\begin{array}{c}\text { fluences } \\
\left(\mathrm{He}^{+} / \mathrm{cm}^{2}\right)\end{array}$ & $\begin{array}{c}\text { non-irradiated } \\
\text { sample }\end{array}$ & $510^{16}$ & $10^{17}$ & $5 \mathrm{keV} 10^{17}$ & $10^{18}$ & $10 \mathrm{keV}$ \\
\hline $\mathrm{O} / \mathrm{Si}^{*}$ & $3.84 \pm 0.11$ & $3.73 \pm 0.13$ & $3.61 \pm 0.20$ & $3.34 \pm 0.35$ & $3.32 \pm 0.25$ & $3.23 \pm 0.51$ \\
$\mathrm{Mg} / \mathrm{Si}^{*}$ & $1.69 \pm 0.06$ & $1.59 \pm 0.08$ & $1.56 \pm 0.13$ & $1.03 \pm 0.17$ & $1.18 \pm 0.27$ & $1.48 \pm 0.23$ \\
\hline \hline
\end{tabular}

${ }^{*}$ Mean value of the ratios \pm the standard deviation calculated for several thicknesses in the region completely amorphized (thickness $\leq 40 \mathrm{~nm}$ ). It should be noted that the composition of the irradiated region may be more altered near the surface (see text for details). In this case, the values in the table for the $10 \mathrm{keV}$ experiment may be underestimated since they do not take into account the deeper amorphized region at $\sim 50-90 \mathrm{~nm}$.

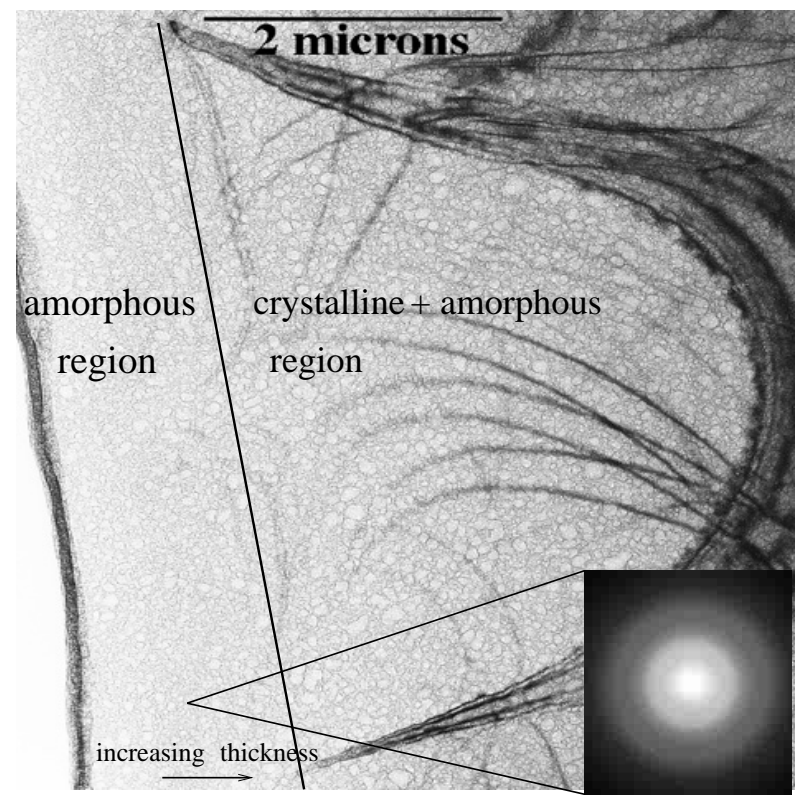

Fig. 2. TEM picture of a crystalline olivine sample irradiated with $10^{18} 10 \mathrm{keV} \mathrm{He} / \mathrm{cm}^{2}$. The dark lines at the right hand side are Bragg diffraction lines. The picture in the bottom right is the electron diffraction pattern of the irradiated sample taken in the amorphous region. The observed diffuse halo is characteristic of an amorphous material. Note the presence of bubbles in the sample

ratios of the sample irradiated at $10 \mathrm{keV}$ as a function of the sample thickness (because iron is much less abundant than magnesium in the sample, we have not measured its evolution under irradiation). Where the sample thickness is smaller than $\sim 90 \mathrm{~nm}$ for the $10 \mathrm{keV}$ experiments ( $\leq 40 \mathrm{~nm}$ at $4 \mathrm{keV}$ ), the sample is completely amorphized. As the thickness increases, the amount of non-irradiated, crystalline, mineral increases and at very large thicknesses the composition is dominated by that of the initial nonirradiated olivine. We observe that the $\mathrm{O} / \mathrm{Si}$ and $\mathrm{Mg} / \mathrm{Si}$ ratios decrease in the ion-transparent region indicating that the stoichiometry of the olivine is modified by the irradiation. Similar trends are observed in the $4 \mathrm{keV}$ experiments. In addition, at $4 \mathrm{keV}$, the decrease in the $\mathrm{O} / \mathrm{Si}$ and $\mathrm{Mg} / \mathrm{Si}$ ratios becomes more pronounced as the irradiation fluence increases (Table 1). It should be noted that the composition of the irradiated regions may not be homogeneous, but may be rather more altered at the surface. Indeed, in

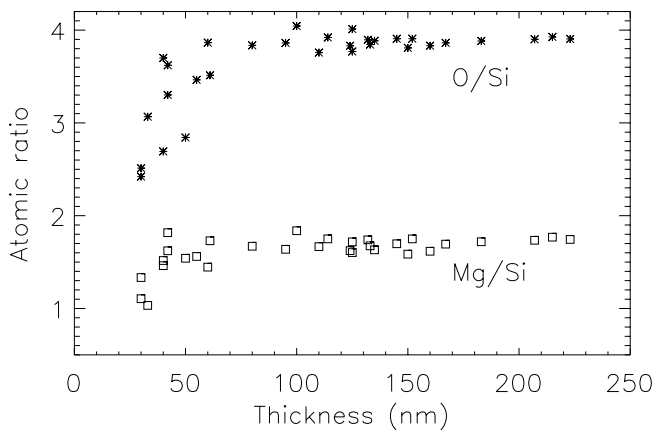

Fig. 3. Evolution of the atomic ratio $\mathrm{O} / \mathrm{Si}$ (stars) and $\mathrm{Mg} / \mathrm{Si}$ (squares) with sample thickness for the $10 \mathrm{keV}$ experiment, $10^{18} \mathrm{ion} / \mathrm{cm}^{2}$. The uncertainty in the thickness $(\sim 20-30 \mathrm{~nm})$ has not be shown for clarity. The uncertainty in the determination of each ratio (not shown) is well-represented by the dispersion of the measurements

the $10 \mathrm{keV}$ experiment, the amorphized region has a thickness of $90 \pm 10 \mathrm{~nm}$, the ratios only decrease for thickness smaller than 50-60 nm (Fig. 3). Two mechanisms could explain the observed stoichiometric changes in the shallower amorphized regions. The elements near the sample surface can be sputtered from the front surface but also from the back surface if the region is thin. In addition, some elements, ionized by electronic collisions and ejected from the lattice by electrostatic repulsion, may diffuse to the surface and be desorbed from the target.

\section{Astrophysical implications}

The $\mathrm{He}^{+}$fluences used in our experiments are compatible with those expected from interstellar shock waves. Indeed, supernova-generated shock waves shock, ionise and accelerate a column of gas of the order of $10^{18}$ $10^{19} \mathrm{H} / \mathrm{cm}^{2}$. This gas collides with the dust grains during the time that they are swept-up by the shock wave. Smaller grains are swept up faster than larger grains, and thus grains are subjected to fluences ranging from $\sim 10^{16}\left(\mathrm{H}, \mathrm{H}^{+}\right) / \mathrm{cm}^{2}$ for $\sim 5 \mathrm{~nm}$ radius-grain to $\sim 10^{18}(\mathrm{H}$, $\left.\mathrm{H}^{+}\right) / \mathrm{cm}^{2}$ for $\sim 250 \mathrm{~nm}$ radius-grain (Jones et al. 1996). The corresponding $\mathrm{He}, \mathrm{He}^{+}$fluences are then $\sim 10^{15}$ to $\sim 10^{17}\left(\mathrm{He}, \mathrm{He}^{+}\right) / \mathrm{cm}^{2}$ for small and large grains, respectively. These experiments show that the irradiation of interstellar silicate grains, during the propagation of interstellar shocks, may efficiently alter the structure and 
chemical composition of the silicate dust. It should be pointed out that in interstellar shock waves, in addition to $\mathrm{He}^{+}$implantation, grains also experience proton implantation, with fluences ten times higher. Proton irradiation of crystalline silicates causes amorphization and changes in their chemical composition (Bradley 1994; Dukes et al. 1999). Although, at a given velocity shock, protons are less energetic than $\mathrm{He}^{+}$ions and will thus cause damage in a thinner layer at the surface of the grains, it is likely that both $\mathrm{H}^{+}$and $\mathrm{He}^{+}$implantation participate efficiently in grain alteration in shocks in the ISM.

Although crystalline silicates are produced around evolved stars, no evidence for their presence in the ISM has been found yet. Our experiments show that the dust grains irradiated in high velocity shocks in the ISM $\left(v_{\mathrm{s}} \geq\right.$ $100 \mathrm{~km} \mathrm{~s}^{-1}$ ) will be efficiently amorphized. With $\mathrm{He}^{+}$ions at 4 and $10 \mathrm{keV}$ respectively (corresponding to $\sim 430$ and $700 \mathrm{~km} \mathrm{~s}^{-1}$ velocity shocks, respectively), a surface layer of thickness of $\sim 40$ and $90 \mathrm{~nm}$ will be amorphized. Adopting an MRN size distribution (Mathis et al. 1977) for the crystalline dust component with $n(a) \propto a^{-3.5}$ and grain sizes from 5 to $250 \mathrm{~nm}$, we find that $\sim 80$ and $95 \%$ of the total crystalline dust volume will be amorphized. However, if grain fragmentation in shocks is an efficient process (Jones et al. 1996) the degree of amorphization will be even higher. This could thus explain the observed absence of crystalline silicates in the ISM.

The irradiation process also alters the compactness of the sample because it creates bubbles of helium (or vacuum) in the sample. The modelling of the silicate bands in the ISM indicates that interstellar grains are porous ( $\sim 25-50 \%$ of porosity, Mathis 1998). This porosity is generally explained by coagulation of dust grains. However, our results suggest that it could also be due to the implantation of ions into the grains in shocks.

Finally, our experiments show that the composition of the irradiated sample is different from the initial sample. As the ion fluence increases, the irradiated layer loses oxygen and magnesium and its composition tends toward a pyroxene-type composition with $\mathrm{O} / \mathrm{Si}=3$ and $\mathrm{Mg} / \mathrm{Si}=1$. This is compatible with the apparent chemical evolution of the silicate dust composition suggested by the fact that young silicates around evolved stars are identified with olivine, whereas around protostars the old silicates are pyroxene-rich (Dorschner et al. 1988; Bowey et al. 1998; Demyk et al. 1999).

\section{Conclusion}

The results of our experiments show that irradiation of interstellar silicate grains in supernova shocks probably plays an important role in their physical and chemical evolution in the ISM. The alteration of the olivine samples, observed in our experiments is compatible with the observed evolution of the interstellar silicate dust, from its birth to its incorporation into protostellar objects. The amorphization of olivine in our experiments suggests that one shock wave $\left(v_{\mathrm{s}} \geq 400 \mathrm{~km} \mathrm{~s}^{-1}\right.$, i.e. $\left.E_{\mathrm{He}^{+}} \geq 4 \mathrm{keV}\right)$ is sufficient to amorphize most crystalline Mg-rich olivine grains in an MRN grain size distribution and also most probably other crystalline silicates. In addition, the evolution of the silicate composition, loss of oxygen and magnesium, under $\mathrm{He}^{+}$irradiation, is consistent with silicate evolution in the ISM, i.e., from young olivine-type silicates around old AGB stars to old pyroxene-type silicate around protostars as observed by ISO. Ion irradiation also induces some porosity into the silicate structure through the formation of bubbles, and porosity seems to be a necessary pre-requisite in the interpretation of observations of interstellar and circumstellar silicates. We are currently following up this work with the characterisation of the infrared spectral characteristic of the irradiated sample, for comparison with observations. Further irradiation experiments (e.g., other silicates, carbonaceous materials) and modelling are planned in order to investigate the consequences of irradiation on the dust evolution in the ISM.

Acknowledgements. We would like to thank O. Kaitasov, S. Gautrot, M. Chaumont, R. Meunier and D. Le Du for having performed the irradiation experiments at the CSNSM in Orsay. Support from the PNP-CNRS is acknowledged.

\section{References}

Borg, J., Chaumont, J., Langevin, Y., et al. 1979, in The ancient sun: Fossil record in the earth, moon and meteorites, Boulder, CO, October 16-19, 431

Borg, J. 1982, Thèse Université de Paris-Sud

Bowey, J. E., Adamson, A. J., \& Whittet, D. C. B. 1998, MNRAS, 298, 131

Bradley, J. P. 1994, Science, 265, 925

Cami, J., De Jong, T., Justtanont, K., et al. 1998, Ap\&SS, 255, 339

Carrez, Ph., Leroux, H, Cordier, P., et al. 2001, submitted to Philos. Mag. A

Day, K. L. 1977, MNRAS, 178, 49

Demyk, K., Jones, A. P., Dartois, E., et al. 1999, A\&A, 349, 267

Demyk, K. 2000, Thèse, Université Paris-Sud

Demyk, K., Dartois, E., Wiesemeyeret, H., et al. 2000, A\&A, 364,170

Dorschner, J., Friedemann, C., Guertler, J., et al. 1988, A\&A, 198,223

Dukes, C. A., Baragiola, R. A., \& McFadden, L. A. 1999, JGR, 104, 1865

Jones, A. P., Tielens, A. G. G. M., \& Hollenbach 1996, ApJ, 469,740

Jones, A. P. 2000, JGR-Space Phys., 105, 10257

Kemper, C., Waters, R., de Jong, T., et al. 2000, in ISO beyond the peak: The second workshop on analytical spectroscopy, 2-4 Feb. 2000, Vilspa

Mathis, J. S. 1998, ApJ, 497, 824

Mathis, J. S., Rumpl, W., \& Nordsieck, K. H. 1977, ApJ, 217, 425

Sogawa, H., \& Kozasa, T. 1999, ApJ, 516, L33

Sylvester, R. J., Kemper, F., Barlow, M. J., et al. 1999, A\&A, 352,587

Waters, L. B. F. M., Beintema, D. A., Zijlstra, A. A., et al. 1998, A\&A, 331, L61 